\title{
Unjamming of Granular Packings due to Local Perturbations: Stability and Decay of Displacements
}

\author{
M. Reza Shaebani, ${ }^{1,2}$ Tamás Unger, ${ }^{1,3}$ and János Kertész ${ }^{1,3}$ \\ ${ }^{1}$ Department of Theoretical Physics, Budapest Univ. of Techn. and Econ., H-1111 Budapest, Hungary \\ ${ }^{2}$ Institute for Advanced Studies in Basic Sciences, Zanjan 45195-1159, Iran \\ ${ }^{3}$ Solid State Research Group of the HAS, Budapest Univ. of Techn. and Econ.
}

(Dated: November 21, 2018)

\begin{abstract}
We study the mechanical response generated by local deformations in jammed packings of rigid disks. Based on discrete element simulations we determine the critical force of the local perturbation that is needed to break the mechanical equilibrium and examine the generated displacement field. Displacements decay as a power law of the distance from the perturbation point. The decay exponent and the critical force exhibit nontrivial dependence on the friction: Both quantities are nonmonotonic and have a sharp maximum at the friction coefficient 0.1 . We find that the mechanical response properties are closely related to the problem of force-indeterminacy where similar nonmonotonic behavior was observed previously. We establish direct connection between the critical force and the ensemble of static force networks.
\end{abstract}

PACS numbers: 45.70.-n,45.70.Mg,45.70.Cc,83.80.Fg Keywords:

Granular materials constitute an ideal field for studying the physics of the jamming transition [1, 2]. One of the most exciting challenges of research is to provide better understanding of the onset of yielding in granular media, an example of unjamming. When the external load on a static assembly of grains is changed at a certain point the load may become incompatible with the inner structure of the packing and the solid state looses its stability. How exactly this happens on grain-scale and what are the key features of the transition between statics and flow are intriguing and unresolved problems [3, 4, , 5, 6]. Moreover, it is of essential importance in many applications to be able to predict, initiate or prevent such transitions.

In this Letter we study yielding induced by local perturbations and focus on two response properties. First, we investigate the resistance of the packing that is exerted against a local deformation. Second, based on the generated displacement field, we study how far the effect of the perturbation penetrates into the packing. In recent years local perturbations were extensively used to study the nature of stress transmission in granular media [7, 8, 9]. These perturbations were typically weak in the sense that they did not break the stability: The original contact network can maintain the perturbation, particle displacements are reversible and occur merely due to elastic distortions of contacts [8]. Here we deal with rigid (undeformable) grains with help of the simulation technique of contact dynamics [10, 11] therefore elastic deformations are excluded. As opposed to the weak perturbations, in our case, plastic deformation of the packing is initiated [3, 4]. Plastic rearrangements caused by local perturbations have been studied in recent experiments [5] where interesting power law decay of the rearrangement field was found.

An important question we address here is what effect the particle-particle friction $\mu$ has on the response of the packings. This was motivated by the prediction that response properties may show nonmonotonic behavior as the function of $\mu$, i.e. small and large frictions lead to similar behavior which is different from the behavior for intermediate friction [12]. This idea was based on the ensemble of admissible force configurations.

It is known that, in general case, mechanical equilibrium and Coulomb condition do not determine contact forces uniquely $[6,9,12,13]$. Consequently there is an ensemble of force networks that satisfy these conditions in the same contact geometry and for the same external load. It depends strongly on friction how large the indeterminacy of individual contact forces is. E.g. for frictionless rigid grains the indeterminacy vanishes and the ensemble shrinks to a single admissible force network 12]. It was found for $2 \mathrm{D}$ packings of disks that indeterminacy of forces becomes the largest for $\mu \approx 0.1$ [12], further increase of the friction leads again to smaller indeterminacy. It was argued in [12] that the extent of force-indeterminacy must affect the stability of the packing, because the more freedom the forces have the more possibility the packing has to resolve changes of the load without rearrangements. This suggests that mechanical response properties may show nonmonotonic dependence on the coefficient of friction. The main message of the present work is that such correlation indeed exists.

The connection between mechanical properties and force indeterminacy was also examined from an other point of view in [14], where the maximum admissible shear tress was deduced from the ensemble of force networks for packings of frictionless soft particles.

The systems we examine are two-dimensional random packings of perfectly rigid disks in zero gravity. The unit of the length is set to the maximum grain radius, radii are distributed uniformly between 0.5 and 1 . The number of the grains contained by the packings ranges from 500 to 8000 . Our numerical experiments consist of two 
parts. First by compression we generate dense random packings then we probe the packings by perturbing single contacts. We apply the contact dynamics method for both procedures.

The compaction starts from a gas-like state with grains randomly distributed in a square-shaped cell. Periodic boundary conditions are applied in both directions and there are no walls in the system. Instead of using pistons the compaction is achieved by the method of Andersen [15]: We impose constant external pressure $p_{\text {ext }}$ and let the volume of the cell evolve in time. A detailed description of the coupling between the pressure bath and the system can be found in [15]. Here we mention only the favorable feature of this method that it gives rise to homogeneous compression and, due to exclusion of side walls, boundary effects are avoided.

As the size of the cell decreases, grains form contacts and start building up the inner pressure in order to avoid interpenetration. Finally a static, jammed configuration is reached where the grains block further compaction. In the final packing contact forces are such that they provide mechanical equilibrium for each grain and the corresponding inner pressure $p_{\text {in }}$ equals to $p_{\text {ext }}$.

After that we turn to the perturbation part where we choose a pair of contacting grains and force them to move apart. One way of doing this would be to apply a small normal force between the two grains and continually increase it. It is expected that if the perturbation force is small enough then it can be resolved by the packing without rearrangements. If the force is increased further the yield point will be reached where the perturbation induce sliding and/or opening of some contacts and initiates collective rearrangements of the particles at least in the vicinity of the perturbation point.

It is very time consuming in computer simulations to scan a region of the perturbation force in order to find the yield point. Therefore we apply another method. Instead of tuning the force we control the deformation. The idea is to bring the system immediately to the yield point by enforcing the opening of the contact: We prescribe a small gap at the contact of perturbation and then determine the force that is needed to fulfill this constraint. This concept is suited very well to the contact dynamics method where interparticle forces are handled as constraint forces, i.e. they are calculated based on constraint conditions which prescribe the relative motion of the contact surfaces [10, 11]. It is beneficial to choose small gap size as we are interested in the onset of motion, how the static structure breaks due to the perturbation. Large deformations, e.g. creation of new contacts, are out of the scope of the present study. We checked that for small gap sizes the displacement field (up to a constant factor) and the critical perturbation force become independent of the size of the gap. Our numerical measurements are performed in this region, the size of the gap $g$ is set to $10^{-9}$.

With the above technique we open up the contact that is selected for the perturbation and measure the gener-

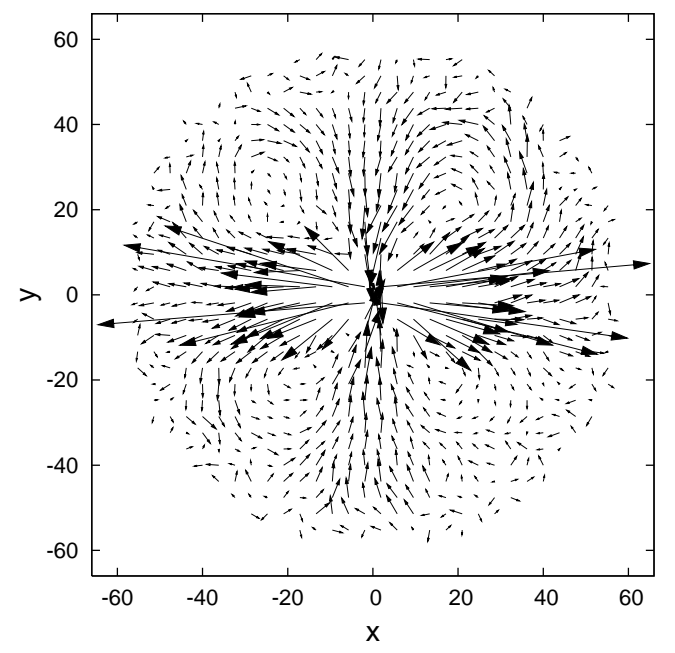

FIG. 1: Displacement response field in the contact frame averaged over several thousand perturbations. The system contained 3000 disks with friction coefficient 0.5.

ated displacement field and the required perturbation force. The latter describes the strength of the system against the local perturbation and we will refer to it as the critical force $F_{\text {crit }}$. Both the force and the displacement response depend strongly on the place of the perturbation. First we check their average properties.

By studying the displacement field our goal is to find out how far the rearrangements have to penetrate into the packing in order to allow the prescribed local deformation. Is there a related length scale? When a single contact is perturbed the displacements of particle centers form a disordered vector field which can be widespread or more localized depending on the perturbed contact. In order to calculate the average displacement field we perturb all contacts one by one always starting with the original static packing. In each case the particle movements are recorded in the local contact frame where the perturbed contact sits in the origin and the $x$-axis is chosen parallel to the contact normal, i.e. $x$ indicates the direction of the separation. Fig. [1]shows a smooth displacement field obtained by averaging over the perturbed contacts. The apparent circular shape of the system is the consequence of the averaging because the original square shape can have different orientations when transformed into different contact frames.

The quadrupolar structure in Fig. 1 is interestingly very similar to the displacement fields that have been observed in sheared systems of deformable frictionless grains [16], where localized quadrupolar deformations appear at the onset of plastic events. Here, for further analysis we consider only the magnitude of the displacement vectors and their distance $r$ from the place of the perturbation (angle of the position is averaged out). The decay of the the average displacement $d$ is shown in Fig. 2

The curves $d(r)$ show power law decay

$$
d \propto r^{-\alpha} .
$$




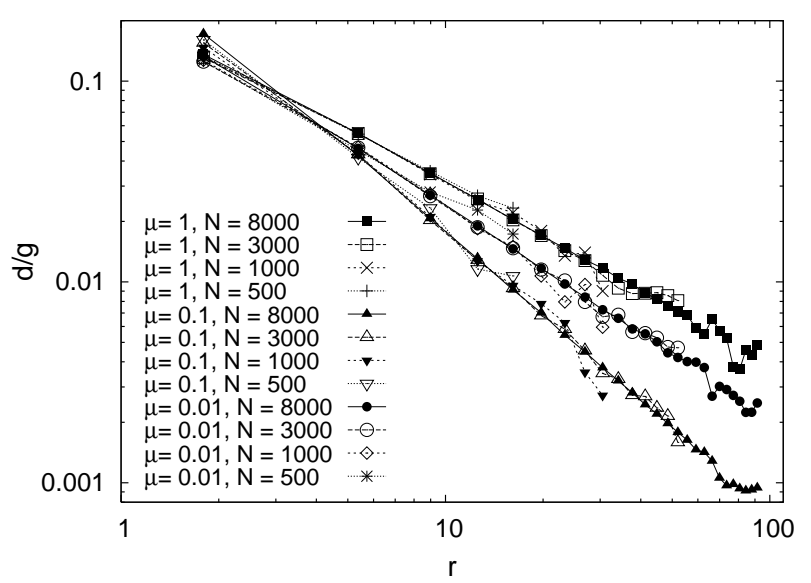

FIG. 2: The magnitude of the displacements, $d$, in terms of the distance from the perturbed contact, $r . g$ stands for the gap generated at the perturbed contact. Different slopes correspond to different friction coefficients $\mu$. For each value of friction four systems of different sizes are investigated. The total number of particles are between 500 and 8000 .

Thus we find that no penetration length can be related to the rearrangements, but instead, the penetration can be best characterized by an exponent $(\alpha)$.

Variation of the system size (Fig. 2), within the modest range allowed by the numerical tools, shows no systematic change in the exponent. There is, however, a strong dependence on friction. The values we obtained for $\alpha$ are between 0.7 and 1.4 [17]. Similar power law behavior with the same range of $\alpha$ has been found experimentally by Kolb et al. [5] by moving an intruder in a system of disks. Next we discuss the influence of friction on the exponent and on the critical force.

It is important to note that the packings, that are subjected to perturbations, are newly generated for each value of friction therefore different values of $\mu$ are accompanied by different packing structures. In Fig. 31a the average contact number $z$ [18] and the average contact force $\left\langle F_{\text {cont }}\right\rangle$ is plotted. In $F_{\text {cont }}$ we take only the normal component of the contact force into account, the average \langle\rangle is meant over all the contacts of the given packing. The contact forces are measured in units $F_{0}$ set by the external pressure and by the average radius of the particles, $F_{0}=2 R_{\text {avg }} P_{\text {ext }}$. The behavior of the packing fraction (not shown) is similar to that of $z$, its value is $0.84(0.80)$ in the low (high) friction limit. Quantities, that describe the properties of the packing, have the common behavior that they exhibit plateaus for low and high friction and show a smooth and monotonic transition in between.

We find a completely different behavior in the response (Fig. 3,b). Both $\alpha$ and the average critical force $\left\langle F_{\text {crit }}\right\rangle$ are nonmonotonic functions of the friction coefficient and exhibit a quite sharp peak at the same place $\mu \approx 0.1$. This is a remarkable behavior because all systems are subjected to exactly the same compaction and perturba-
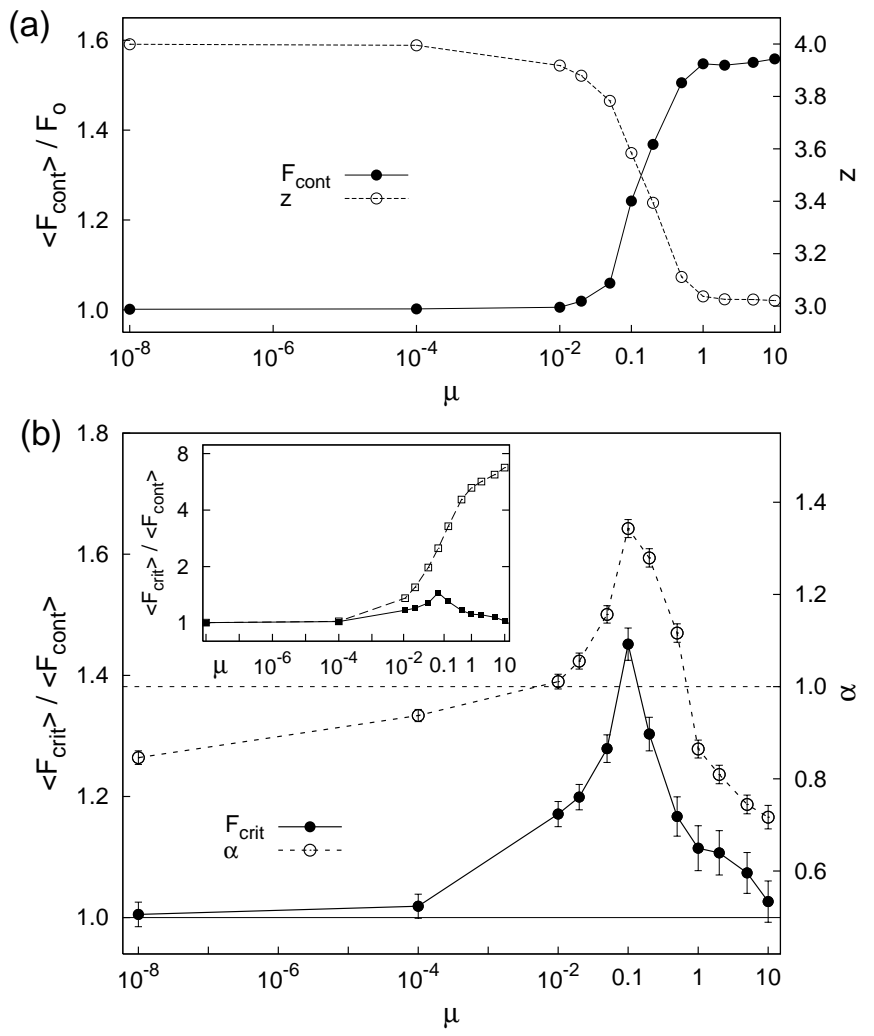

FIG. 3: (a) Influence of friction on the average normal contact force $\left\langle F_{\text {cont }}\right\rangle$ (full circles) and on the contact number z (open circles). (b) Average critical force $\left\langle F_{\text {crit }}\right\rangle$ (full circles) with respect to the average normal contact force and the penetration exponent $\alpha$ (open circles) as functions of the friction coefficient $\mu$. The horizontal lines show the reference value 1 for the exponent $\alpha$ (dashed) and for the critical force (solid). The inset shows the role of the structural change in the packing. For normally constructed packings $\left\langle F_{\text {crit }}\right\rangle$ is plotted with full squares (new packing is constructed for each friction). Open squares were obtained by changing the friction in a fixed packing configuration.

tion procedures, friction was the only quantity that has been changed.

When the friction is increased starting from zero, packings are getting stronger against the perturbation and the induced rearrangements become more localized. At $\mu \approx 0.1$ the process takes a sharp turn and further increase of the friction leads to softening and delocalization.

The nonmonotonic behavior and the position of the maxima in (Fig. 3.,b) are reminiscent of the forceindeterminacy that was reported in [12]. In order to clarify what role the force-indeterminacy plays in the mechanical response we perform two independent numerical measurements in the same test system: on the one hand we investigate the force-indeterminacy on the other hand we determine the critical forces. Then the data are compared on the level of single contacts.

As mentioned before, in frictional case contact forces are not determined uniquely but there is an ensemble 


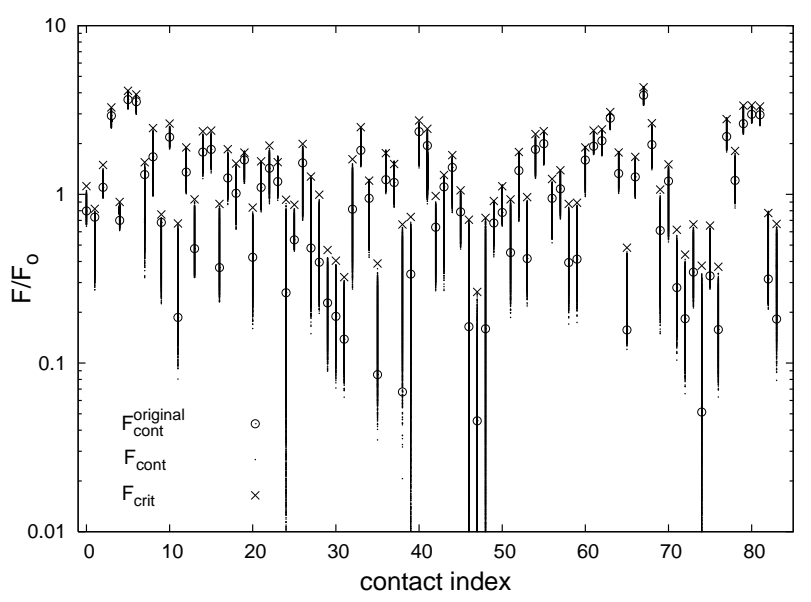

FIG. 4: The critical force $F_{\text {crit }}$ (cross), the original contact force (open circle) and the possible values of the normal contact forces (dots, mostly merged to intervals) are shown for each contact of the packing.

of force networks that correspond to equilibrium and to the compatibility conditions (forces are limited by the Coulomb cone). This ensemble of force networks forms a convex set in the space of the contact forces [12]. We explore this high dimensional solution set using a random walk starting with the original force network. The exploration process is the same as in [12]. Each step of the random walk provides one possible force-network. Because the dimension of the solution set is large (proportional to the number of contacts) only small systems can be handled efficiently. We generate a packing of 50 disks with $\mu=0.1$ (total number of contacts is 85 ). As a consequence of the convexity, the possible values of the normal (the tangential) component of any contact force are defined by an interval. These intervals are traced out in Fig. 4 with help of 20000 force networks that are collected by the exploration procedure.

The figure shows also the original contact forces (pro- vided by the compaction process) and the critical forces. The data reveal that the critical force obtained by the perturbation coincides with the maximum of the equilibrium solutions at each contact. Thus the critical force can be directly obtained from the force-ensemble. It also can be seen that a strong (weak) contact has typically large (small) critical force.

This picture also explains the limit of small and large frictions where the indeterminacy vanishes. In these cases the length of the force-intervals goes to zero. Which means that a pair of contacting particles can not resist a force of separation larger than the force itself that originally presses the two contact surfaces together. This is why the average critical force approaches $\left\langle F_{\text {cont }}\right\rangle$ on the left and right side of Fig. 3. b. $\left\langle F_{\text {crit }}\right\rangle$ can differ significantly from $\left\langle F_{\text {cont }}\right\rangle$ only if the indeterminacy of forces is large in the packing.

It was argued in [12] that the nonmonotonic forceindeterminacy can be attributed to two competing effect of the increasing friction: first, the strengthening of the contacts (increase), and second, the structural change inside the packing (decrease). Accordingly, if the second effect is switched off the decay part of the curve $F_{\text {crit }}(\mu)$ disappears as shown by the inset of Fig. 3 b. Here the response of the same packing configuration was tested for various values of friction.

In this Letter we reported nontrivial dependence of the mechanical response on the coefficient of friction and related the results to the indeterminacy of forces. Nonetheless, many questions arise that urge further studies. We list three of them here. What is the origin of the displacement exponent $\alpha$ ? Numerical data show strong correlation between $\alpha$ and the critical force. What is the connection between them? Finally, how does the picture, which is presented here for rigid particles, change for deformable ones?

We acknowledge support by grant OTKA T049403 and Öveges project GranKJ06 of KrI and DTKKTH.
[1] A. J. Liu and S. R. Nagel, eds., Jamming and Rheology : Constrained Dynamics on Microscopic and Macroscopic Scales (CRC, 2001).

[2] T. S. Majmudar, M. Sperl, S. Luding, and R. P. Behringer, Phys. Rev. Lett. 98, 058001 (2007).

[3] J. N. Roux, Phys. Rev. E 61, 6802 (2000).

[4] G. Combe and J. N. Roux, Phys. Rev. Lett. 85, 3628 (2000).

[5] E. Kolb, C. Goldenberg, S. Inagaki, and E. Clement, J. Stat. Mech. p. P07017 (2006).

[6] S. McNamara, R. Garcia-Rojo, and H. Herrmann, Phys. Rev. E 72, 021304 (2005).

[7] J. Geng, E. Longhi, R. P. Behringer, and D. W. Howell, Phys. Rev. E 64, 060301 (2001).

[8] C. Goldenberg and I. Goldhirsch, Nature 435, 188 (2005).
[9] S. Ostojic and D. Panja, Phys. Rev. Lett. 97, 208001 (2006).

[10] M. Jean, Comput. Methods Appl. Mech. Engrg. 177, 235 (1999).

[11] L. Brendel, T. Unger, and D. E. Wolf, in The Physics of Granular Media (Wiley-VCH, Weinheim, 2004), pp. 325-343.

[12] T. Unger, J. Kertész, and D. E. Wolf, Phys. Rev. Lett. 94, 178001 (2005).

[13] J. H. Snoeijer, T. J. H. Vlugt, M. van Hecke, and W. van Saarloos, Phys. Rev. Lett. 92, 054302 (2004).

[14] J. H. Snoeijer, W. G. Ellenbroek, T. J. H. Vlugt, and M. van Hecke, Phys. Rev. Lett. 96, 098001 (2006).

[15] H. C. Anderson, J. Chem. Phys. 72, 2384 (1980).

[16] C. E. Maloney and A. Lemaitre, Phys. Rev. E 74, 016118 (2006). 
[17] For comparison, the exponent 1 corresponds to a two dimensional incompressible fluid perturbed by a localized volume injection.
[18] Rattlers (particles without force-carrying contacts) are disregarded in $z$. 